\title{
Efeitos farmacológicos do extrato aquoso de Solidago chilensis Meyen em camundongos
}

\author{
ASSINI, F.L. ${ }^{*}$; FABRÍCIO, E.J1.; LANG, K.L¹. \\ 1Universidade do Contestado, CEP:89700-000 Concórdia-Brasil *fabricioassini@ hotmail.com
}

\begin{abstract}
RESUMO: Solidago chilensis Meyen (Asteraceae) é uma espécie nativa da América do Sul (Brasil) encontrada principalmente na região Sul do Brasil onde é conhecida popularmente como arnica-do-mato. Na medicina popular, ela é utilizada como diurética, cicatrizante, e antiinflamatória. No presente trabalho, os efeitos farmacológicos do extrato aquoso das raízes de S. chilensis foram avaliados em modelos experimentais in vivo de atividade tipo-antidepressiva, antiinflamatória, antinociceptiva, e locomotora. O extrato $\left(25,100\right.$ e $\left.250 \mathrm{mg} \mathrm{kg}^{-1}\right)$ foi administrado por via oral 30 min antes dos experimentos comportamentais. Os resultados mostram que, nas doses utilizadas, o extrato aquoso de S. chilensis não apresentou atividade tipo-antidepressiva apesar de induzir efeitos analgésico e antiinflamatório significativos. Uma redução da atividade locomotora foi observada com a maior dose $\left(250 \mathrm{mg} \mathrm{kg}^{-1}\right)$ administrada, sugerindo efeito sobre o sistema nervoso central. Em conclusão, os resultados estão de acordo com a literatura acerca dos efeitos analgésicos e antiinflamatórios da planta, sugerindo também uma atividade do extrato de $S$. chilensis sobre o sistema nervoso central. Essas observações, porém, não excluem um possível efeito relaxante muscular periférico do extrato.
\end{abstract}

Palavras-chave: Solidago chilensis, analgesia, inflamação, depressão, atividade farmacológica

\begin{abstract}
Pharmacological effect of aqueous extract from Solidago chilensis Meyen on mice. Solidago chilensis Meyen (Asteraceae) is a species native to South America (Brazil), found especially in the south region of Brazil, where it is commonly known as "arnica-do-mato". In folk medicine, it has been used as diuretic, healing and anti-inûammatory. In the present study, the pharmacological effects of aqueous extracts from roots of $S$. chilensis were assessed in vivo in experimental models for antidepressant, anti-inflammatory and locomotor-type activity. The extract $\left(25,50\right.$ and $\left.250 \mathrm{mg} \mathrm{kg}^{-1}\right)$ was administered by the oral route 30 minutes prior to behavioral tests. Results indicate that, at the employed levels, aqueous extract from $S$. chilensis did not show antidepressant-type activity although it induced significant analgesic and anti-inflammatory effects. A reduction in the locomotor activity was noted at the highest administered level (250 $\mathrm{mg} \mathrm{kg}^{-1}$ ), suggesting an effect on the central nervous system. In conclusion, results agree with the literature about analgesic and anti-inflammatory effects of this plant, also suggesting an activity of $S$. chilensis extract on the central nervous system. These remarks, however, do not exclude a possible peripheral muscular relaxing effect of the extract.
\end{abstract}

Key words: Solidago chilensis analgesia, inflammation, depression, pharmacological activity

\section{INTRODUÇÃO}

O gênero Solidago (Asteraceae) abrange cerca de 120 espécies distribuídas por todo o mundo, sendo amplamente utilizadas na medicina tradicional de vários países, principalmente no tratamento de problemas inflamatórios e como diuréticas (Blumenthal et al.,1998). Na América do sul, a espécie nativa é Solidago chilensis Meyen, conhecida popularmente como erva-lanceta, arnicabrasileira ou arnica-silvestre. A espécie vem sendo amplamente utilizada no Brasil, principalmente na forma de extratos alcoólicos e infusões, sendo associada às propriedades anti-sépticas, analgésicas, cicatrizantes e antiinflamatórias (Pio Corrêa, 1984; Mors et al., 2000; Lorenzi \& Matos, 2002).

Quanto à composição química de plantas do gênero Solidago é descrita a presença de terpenos, saponinas, ácidos fenólicos e altas quantidades de 
flavonóides, principalmente quercetina, campferol e rutina (Lorenzi \& Matos, 2002). No entanto, são poucos os estudos relativos à composição química de $S$. chilensis. Das raízes dessa espécie já foram isolados diterpenos do tipo labdano (SchmedaHirschmann, 1987) além dos flavonóides quercetina e quercitrina, esse último relatado como constituinte majoritário das partes aéreas da planta (Gutierrez et al., 1981).

A validação etnofarmacológica dos efeitos antiinflamatórios vem sendo realizada nos últimos anos, com atividade dos extratos aquosos e hidroalcoólico sendo descritas no teste da pleurisia (Goulart et al., 2007) e no edema de orelha (Tamura et al., 2009), respectivamente. Estes efeitos antiinflamatórios têm sido associados aos flavonóides, principalmente a quercetina, presentes em ambos os extratos.

É importante salientar que outras plantas ricas em quercetina têm apresentado importante atividade sobre o sistema nervoso central (SNC). Por exemplo, extratos de Hipericum perfuratum, espécie que tem sido amplamente utilizada como antidepressivo, contém altas concentrações de quercetina (Paulke et al., 2006). Além disso, alimentos ricos em quercetina, como o alho, também apresentam efeito tipo-antidepressivo quando testados em modelos animais (Sakakibara et al., 2008).

Desta forma, uma vez que o extrato de $S$. chilensis tem apresentado atividade antiinflamatória em diferentes modelos, mas não possui atividade sobre o SNC validada, este trabalho tem por objetivo avaliar as ações centrais do extrato aquoso de $S$. chilensis em camundongos.

\section{MATERIAL E MÉTODOS}

\section{Material botânico}

Raízes de S. chilenis Meyen foram coletadas em Caibi, Santa Catarina, Brasil e identificadas pelo botânico Prof. Dr. Daniel Falkenberg, da Universidade Federal de Santa Catarina, Florianópolis, SC, Brasil (FLOR34674).

\section{Obtenção do extrato}

As raízes de $S$. chilensis foram secas à temperatura ambiente, em estufa de ar circulante, por 7 dias. Para obtenção do extrato, as raízes foram pulverizadas e submetidas à infusão utilizando água quente a $90^{\circ} \mathrm{C}$ (planta: solvente $1: 10, \mathrm{~m} / \mathrm{v}$ ) por 10 minutos. $O$ infuso foi filtrado e liofilizado (Edward ${ }^{\circledR}$ E-C Micromodulyo Freezer Dryer, USA), com rendimento final de $12 \%$.

\section{Animais}

Foram utilizados camundongos Swiss de ambos os sexos (30-40g), mantidos em gaiolas plásticas em ambiente com temperatura $\left(23 \pm 2^{\circ} \mathrm{C}\right)$ e umidade $(60-80 \%)$ controladas, ciclo claroescuro de 12 horas e comida e água ad libitum. Os protocolos experimentais foram aprovados pelo Comitê de Ética e Pesquisa em Animais número $508 / 08$ e conduzidos de acordo com as diretrizes éticas proposto pela "International Association for Study of Pain" (IASP) (Zimmermann, 1983).

\section{Testes farmacológicos}

Teste da formalina (Dubuisson \& Dennis, 1977): Os animais foram tratados com extrato aquoso de $S$. chilensis Meyen (25, 100 e $250 \mathrm{mg}$ $\mathrm{kg}^{-1} ;$ v.o. $\mathrm{n}=8$ ) ou com igual volume de solução salina $0,9 \%$ e 30 min após cada tratamento receberam a administração intraplantar de formalina 2\% (20 $\mu \mathrm{l}$ animal $^{-1}$ ) na pata posterior direita. O tempo em segundos (s) que cada animal despendeu lambendo a pata tratada com formalina foi medido em dois períodos: de 0 a 5 min (fase 1) e dos 15 aos 30 min finais (fase 2). Este modelo experimental de nocicepção permite distinguir duas fases da resposta nociceptiva. A primeira fase, que é de origem neurogênica (decorrente da estimulação direta dos nociceptores), ocorre nos primeiros 5 min após injeção intraplantar de formalina na pata posterior direita em camundongos. Já a segunda fase, chamada de fase inflamatória (pois depende da liberação de múltiplos mediadores inflamatórios), é observada somente a partir de 15 min após a injeção de formalina e estende-se até 30 minutos. (Hunskaar and Hole, 1987).

Teste do campo aberto (Rodrigues et al., 2002): Após os mesmos tratamentos e intervalos de tempo utilizados nos testes anteriores, novos grupos de animais foram colocados no aparato de campo aberto e registrou-se o número de cruzamentos atravessados pelo animal durante os 5 minutos do teste.

Teste do nado forçado (Porsolt et al., 1978): Após 30 min do tratamento com o extrato aquoso do S. chilensis Meyen (25, 100 e $250 \mathrm{mg} \mathrm{kg}^{-1}$; v.o. $\mathrm{n}=8$ ) ou solução salina $0,9 \%$, os animais foram colocados individualmente em cilindro contendo $23 \mathrm{~cm}$ de água mantida a $25 \pm 1{ }^{\circ} \mathrm{C}$ em sessão única de nado forçado por 5 minutos. Registrou-se o tempo (s) em que cada animal permaneceu imóvel durante o teste, trocando-se a água do recipiente entre os testes de cada animal para evitar que pistas olfativas interferissem no resultado final.

\section{Análise estatística}

Todos os resultados foram apresentados como média \pm EPM. Os dados foram analisados com ANOVA de uma via seguida do post-hoc de Dunnett. Em todas as análises valores de $p<0,05$ foram considerados significativos. 


\section{RESULTADOS}

Na Figura 1 são mostrados os efeitos da administração oral de $S$. chilensis sobre o tempo de reação dos animais aos estímulos nociceptivo e inflamatório quantificados nas duas fases do teste da formalina. A ANOVA de uma via indicou efeito significativo do tratamento tanto na 1 a $[F(3,28)=26,7$; $p=0,0001]$ quanto na 2 a fase $[F(3,28)=140,3$; $p=0,0001]$ do teste. A análise post - hoc demonstrou que todas as doses testadas reduzem o tempo de lambida de pata nas duas fases do teste.

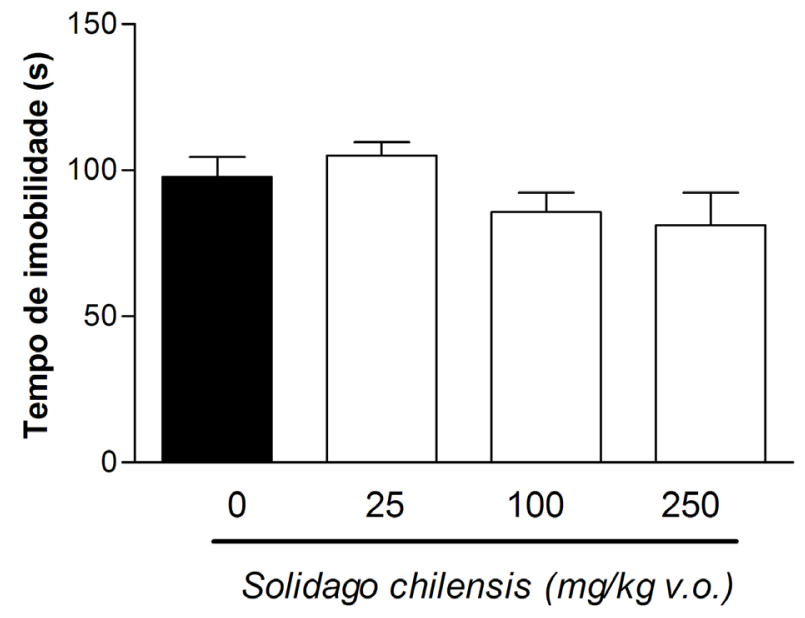

FIGURA 1. Efeitos do extrato aquoso de $S$. chilensis administrado $30 \mathrm{~min}$ antes do teste da formalina. Valores expressos como média \pm EPM $(n=8$ por grupo). * Indica diferença significativa após ANOVA seguida do teste de Dunnett $(p<0,05)$.

extrato de S. chilensis diminuiu significativamente a atividade locomotora dos animais (ANOVA de uma via: $[F(3,28)=6,41 p<0,0001]$. O teste de post - hoc de Dunnett mostra que o efeito hipolocomotor foi significativo apenas após a administração da maior dose $\left(250 \mathrm{mg} \mathrm{kg}^{-1}\right)$.

Os efeitos do tratamento com as diferentes doses do extrato de S. chilensis sobre o tempo de imobilidade no teste do nado forçado são observados na Figura 3. A análise por ANOVA de uma via não detectou efeito significativo para nenhuma das doses testadas $[F(3,28)=2,01 ; p=0,13]$.

\section{DISCUSSÃO}

No presente trabalho foram avaliados os efeitos antidepressivo, antinociceptivo, antiinflamatório e locomotor produzidos pela administração oral do extrato aquoso de $S$. chilensis ( 25 a $250 \mathrm{mg} \mathrm{kg}^{-1}$ ) em camundongos. Os
Fase 1

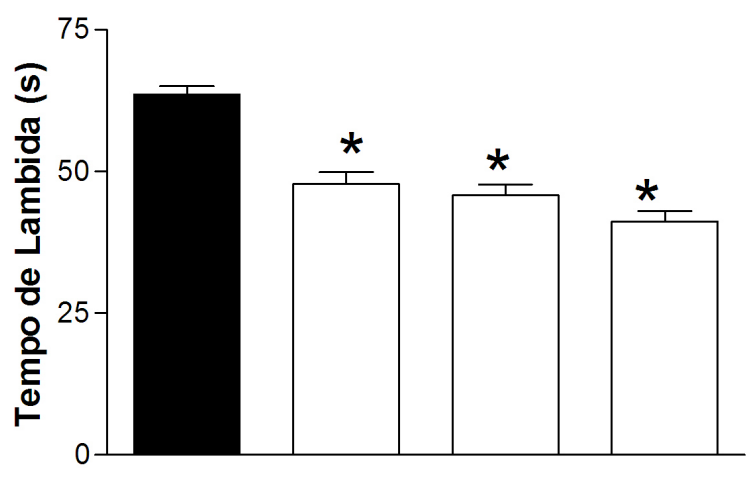

Fase 2

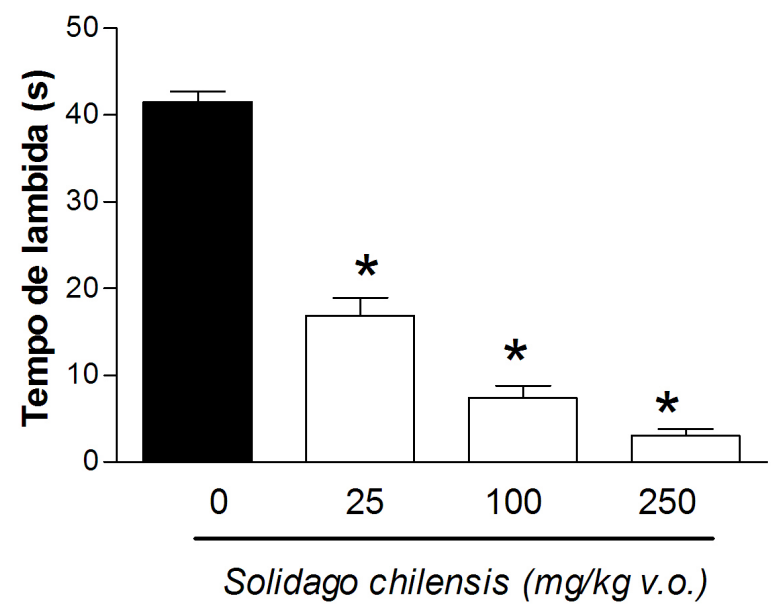

FIGURA 2. Efeito do tratamento com o extrato aquoso de Solidago chilensis Meyen (25, 100 e $250 \mathrm{mg} \mathrm{kg}^{-1}$ v.o.) sobre a atividade locomotora registrada no campo aberto. Os resultados estão expressos como média $\pm \mathrm{EPM}, \mathrm{n}=8$. * Indica diferença significativa após ANOVA seguida do teste de Dunnett $(p<0,05)$.

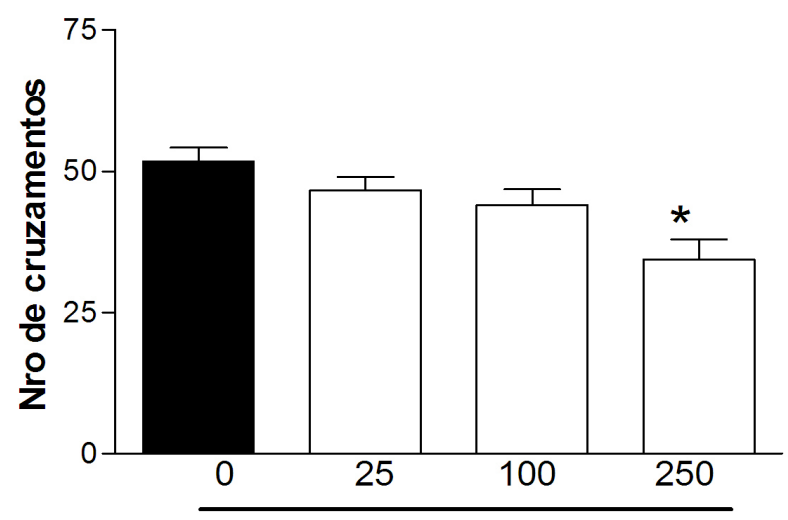

Solidago chilensis ( $m g / k g$ v.o.)

FIGURA 3. Efeitos do extrato aquoso de $S$. chilensis Meyen (25, 100 e $250 \mathrm{mg} \mathrm{kg}^{-1}$ v.o.) administrado 30 minutos antes do teste do nado forçado. Os valores estão expressos como média \pm EPM $(n=8$ animais por grupo). 
resultados não mostram efeito do tipo antidepressivo nos animais tratados com diferentes doses do extrato e submetidos ao teste do nado forçado. Diferentemente, todas as doses utilizadas do extrato apresentaram efeito antinociceptivo, antiinflamatório, bem como hipolocomotor.

Apesar do resultado negativo, cabe ressaltar que este é o primeiro trabalho a verificar a possível atividade antidepressiva da administração aguda do extrato aquoso de $S$. chilensis em camundongos, utilizando para isto o teste do nado forçado (Porsolt et al., 1978). Este teste é amplamente utilizado para a validação farmacológica de fármacos com ação do tipoantidepressiva. Embora o extrato de S. chilensis apresente constituintes também descritos em outros extratos com ação antidepressiva, como o Hipericum perforatum (Paulke et al., 2006) e o pó de Allium cepa (Sakakibara et al., 2008), nas doses utilizadas não foi observada redução significativa no tempo de imobilidade, comportamento que seria sugestivo de efeito antidepressivo (Porsolt et al., 1978). Entre as possíveis hipóteses levantadas para a falta de efeito do extrato, questionou-se a faixa de doses testadas, ou ainda o intervalo de tempo entre a administração e o teste.

Para descartar algumas destas suposições, optou-se por corroborar a já demonstrada ação antiinflamatória do extrato de S. chilensis. Para isso utilizou-se o teste da formalina, o qual é um modelo composto de duas fases. A primeira fase é atribuída ao efeito imediato da formalina em fibras nociceptivas (Abbadie et al., 1997), enquanto a segunda fase representa a dor inflamatória (Hunskaar \& Hole, 1987). Fármacos antiinflamatórios convencionais reduzem a resposta dos animais apenas na segunda fase do teste, enquanto que os analgésicos opióides são eficazes em ambas as fases (Hunskaar \& Hole, 1987). Neste estudo, o extrato aquoso da S. chilensis apresentou eficácia em ambas as fases do teste sugerindo um efeito antinociceptivo e antiinflamatório. Esses dados corroboram as observações de Tamura et al. (2009) que descreveram a redução do edema de orelha em ratos tratados com o extrato hidroalcoólico de $S$. chilensis. Além disso, os resultados observados reforçam o efeito antiinflamatório do extrato aquoso desta planta, já observados por Goulart et al. (2007) no modelo da pleurisia induzida por carragenina. É interessante notar que o presente estudo demonstra pela primeira vez os efeitos do extrato aquoso de $S$. chilensis no teste da formalina.

Para descartar a possível baixa permeabilidade hematoencefálica dos constituintes do extrato, os animais foram submetidos ao teste do campo aberto, o qual é utilizado para avaliar atividade locomotora e é sensível tanto a drogas estimulantes quanto depressoras do SNC (Prut \& Belzung, 2003). Observou-se que a maior dose de $S$. chilensis $\left(250 \mathrm{mg} \mathrm{kg}^{-1}\right)$ exerceu significativa depressão locomotora dos animais até então pouco descrita, sugerindo que a faixa de doses testadas estava dentro de valores razoáveis e que possivelmente os efeitos da maior dose do teste da formalina possam ter sido influenciados pela ação hipolocomotora do extrato. Além disso, apenas com este grupo experimental não podemos descartar uma possível ação sedativa ou relaxante muscular periférica na dose testada.

Por fim, este estudo mostra que apesar de possuir atividade no SNC, o extrato aquoso de $S$. chilensis não apresentou efeito tipo-antidepressivo nas doses testadas e no modelo animal utilizado, possivelmente porque outros compostos com ação central não estavam presentes em concentração suficiente para exacerbar estes efeitos. Observados em conjunto, o pronunciado efeito antiinflamatório e a ação no sistema nervoso central indicada nesse trabalho sugerem o potencial ainda não explorado deste extrato no tratamento de patologia associadas à inflamação no sistema nervoso central, como a doença de Alzheimer, por exemplo (Eikelenboom \& Gool, 2004). Por outro lado, será necessário descartar uma possível atividade relaxante muscular periférica do extrato.

\section{REFERÊNCIA}

ABBADIE, C. et al. Differential contribution of the two phases of the formalin test to the pattern of c-fos expression in the rat spinal cord: studies with remifentanil and lidocaine. Pain, v.69, n.1-2, p.101-10, 1997.

BLUMENTHAL, M.; BUSSE, W.R.; GOLDBERG, A. The complete German commission e monographs. Boston: American Botanical Council, Austin and Integrative Medicine Communications, 1998. 685p.

DUBUISSON, D.; DENNIS, S.G. The formalin test: a quantitative study of the analgesic affects of morphine, meperidine, and brain stem stimulation in rats and cats. Pain, v.4, n.1, p.161-74, 1977.

EIKELENBOOM, P.; VAN GOOL, W.A. Neuroinflammatory perspectives on the two faces of Alzheimer's disease. Journal of Neural Transmission, v.111, n.3, p.281-94, 2004.

GUTIERREZ, A.B.; OBERTI, J.C.; JULIANI, H.R. Constituents of Solidago chilensis (Compositae). Anales de la Asociacion Quimica Argentina, v.69, n.1, p.2731, 1981.

GOULART, S. et al. Anti-inflammatory evaluation of Solidago chilensis in a murine model of pleurisy. Journal of Ethnopharmacology, v.113, n.2, p.346-53, 2007.

HUNSKAAR, S.; HOLE, K. The formalin test in mice dissociation between inflammatory pain. Pain, v.30, n.1, p.103-14, 1987.

LORENZI, H.; MATOS, F.J.A. Plantas medicinais no

Rev. Bras. PI. Med., Botucatu, v.15, n.1, p.130-134, 2013. 
Brasil - nativas e exóticas: Solidago chilensis Meyen. São Paulo: Instituto Plantarum de Estudos da Flora. 2002. 450p.

MORS, W.B.; RIZZINI, C.T.; PEREIRA, N.A. Medicinal plants of Brazil. Reference Publications, Algonac, 2000, $501 \mathrm{p}$.

PAULKE, A.; SCHUBERT-ZSILAVECZ, M.; WURGLICS, M. Determination of St. John's wort flavonoid - metabolites in rat brain through high performance liquid chromatography coupled with fluorescence detection. Journal of Chromatography B, v. 832, n.1, p.109-13, 2006. PIO CORRÊA, M. Dicionário das plantas úteis do Brasil e das exóticas cultivadas. Rio de Janeiro: Impressora Nacional. 1984, 4324p.

PORSOLT, R.D. et al. Behavioral despair in rats: a new model sensitive to antidepressant treatments. European Journal of Pharmacology, v.47, n.4, p.379-91, 1978. PRUT, L.; BELZUNG, C. The open field as a paradigm to measure the effects of drugs on anxiety-like behaviors: a review. European Journal of Pharmacology, v28, n.1, p.3-33, 2003.

RODRIGUES, A.L. et al. Involvement of monoaminergic system in the antidepressant - Like effect of the hydroalcoholic extract of Siphocampylus verticillatus. Life Science, v.27, n.14, p.1347-58, 2002.

SAKAKIBARA, $H$. et al. Antidepressant - Like effect of onion (Alliun cepa L.) powder in a rat behavioral model of depression. Bioscience Biotechnology Biochemistry, v.72, n.1, p.94-100, 2008.

SCHMEDA-HIRSCHMANN, G. A labdan diterpene from Solidago chilensis roots. Planta Médica, v.2, n.2, p.17980, 1987.

TAMURA, E.K. et al. Inhibitory effects of Solidago chilensis Meyen hydroalcoholic extract on acute inflammation. Journal of Ethnopharmacology, v.122, n.3, p.478-85, 2009.

ZIMMERMANN, M. Ethical guidelines for investigations of experimental pain in conscious animals. Pain, v.16, n.2, p.109-10, 1983. 